\title{
Novel Techniques for Improving Anti-Cancer Efficacy via Synergistic Phototherapy
}

\author{
Jui Teng Lin ${ }^{1 *}$, Kuo Ti Chen ${ }^{2}$ and Hsia Wei Liu ${ }^{2,3}$ \\ ${ }^{1}$ New Vision Inc Taipei, Taiwan \\ ${ }^{2}$ Graduate Institute of Applied Science and Engineering, Fu Jen Catholic University, Taiwan \\ ${ }^{3}$ Department of Life Science, Fu Jen Catholic University, Taiwan
}

Received: 阱 March 16, 2018; Published: 阱 March 26, 2018

*Corresponding author: Jui-Teng Lin, New Vision Inc. Taipei, Taiwan, Email: jtlin55@gmail.com

\begin{abstract}
Factors influencing the cancer therapy efficiency in both photothermal therapy (PTT) and photodynamic therapy (PDT) using nanogold particles and photosensitizers, respectively, are analyzed. In PTT, heat diffusion kinetics is used to calculate the temperature increase resulted from the nanogold absorption of light energy, whereas photochemical kinetics is used to find the efficacy of PDT, or the generation rate of reactive oxygen species. Efficacy of cancer therapy may be enhanced by combining PTT and PDT either activated by one light or two lights. For maximum PTT/PDT synergistic efficacy, the concentration of photosensitizers and nanogold required optimization, besides the wavelength of the light matching the absorption peak of PS and nanogold, and the sequential order of PTT and PDT process. External supply of either photosensitizers or oxygen concentration will significantly improve the anti-cancer efficacy via type-II PDT. Optimization is required for maximum synergic efficacy.
\end{abstract}

Keywords: Photothermal Therapy; Optimal; Synergistic effect; Modeling; Heat diffusion; Photochemical kinetics

Abbreviations: PTT: Photothermal Therapy; PDT: Photodynamic Therapy; IND: Investigational New Drug; NRI: Near-Infrared; GNR: Gold Nanorod; PS: Photosensitizers; MB: Methylene Blue

\section{Introduction}

Various methods/technologies have been investigated for the improvement of phototherapy of cancers, including nanomedicine, nano-particles and synergic method combining photothermal therapy (PTT) and photodynamic therapy (PDT) using nanogold particles and photosensitizers, respectively [110]. Cancer or tumor cells death may be caused by photothermal ablation, mechanical damage, and increase in the localized drug concentration. Gold nanoparticles are promising agents for cancer therapy, drug carriers, photo-thermal agents and contrast agents. The U.S. FDA has approved numerous Investigational New Drug (IND) applications for nano-formulations, enabling clinical trials for breast, gynecological, solid tumor, lung, mesenchymal tissue, lymphoma, central nervous system and genito-urinary cancer treatments. Comparing to the visible light, near-infrared (NIR) light offers the advantages of larger absorption and scattering cross sections and much deeper penetration depth in tissues [5-8].

The red-shift of the absorption peak in nanorods is governed by the aspect ratio (defined by as the ratio of the length to the crosssectional diameter), whereas it is governed by the shell thickness in nanoshells [9].

Recent studies have shown that gold nanorods conjugated to antibodies [11-13] could be used for selective and efficient photothermal therapy. Lin et al. [4] proposed the use of a diode laser system having multiple wavelengths for more efficient treatment of cancer tumor. Overheating on the surface area of targeted tissues is always an issue to be overcome. In addition, the distribution of the gold nanorod (GNR) aspect ratios and their concentrations inside the cancer tissues or tumors are also difficult to be controlled for perfectly matching the laser peak absorption. To overcome the penetration issue, Lin et al proposed the use of a train-pulse to increase the volume temperature increase [4] which is particularly useful to larger volume tumors, unless an inserting fiber is used 
to deliver the laser energy. New synergistic treatment modalities combining PDT with PTT could overcome current limitations of PDT, thus achieving enhanced anticancer efficacy. To promote the tumor accumulation of photosensitizers (PS) and to generate heat for synergistic PDT/PTT [10], surface conjugation of PS on nanoparticles has been proposed, which however, has limitations including relatively low loading capacity and the possible leakage of PSs coupled on nanoparticle surfaces during their circulation in biological systems.

In this study, we will review the factors influencing the cancer therapy efficiency in both PTT and PDT using nanogold particles and photosensitizers, respectively. In PTT, heat diffusion kinetics is used to calculate the temperature increase resulted from the nanogold absorption of light energy, whereas photochemical kinetics is used to find the efficacy of PDT, or the generation rate of reactive oxygen species. Besides a review, the measured data of synergistic PDT/PTT [10] will be discussed. We will also present new optimal parameters for maximum PDT efficacy.

\section{Methods and Discussions}

\section{The Modeling System}

\section{Light-1 (for PTT)}

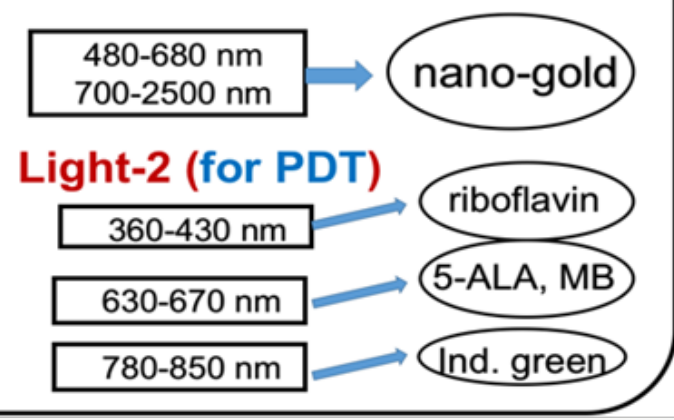

Figure 1: Combined PTT and PTT processes using various lights having wavelength from UV to IR with associate nanogold shapes and photosensitizer [2].

As shown in Figure 1, the tumor cells killing efficiency may be enhanced by combining PTT and PDT using two light sources (either lasers or LED sources), in which the treated tumor tissue is injected by both nanogold solution and photosensitizers [2]. Depending on the types of photosensitizers and the shapes of the nanogold, the light wavelengths matching the absorption may vary from UV, visible to near IR (NIR). For examples, nanosphere absorbs visible light (at 480-680 nm), nanotubes (700-900 nm), nanorod $(700-2500 \mathrm{~nm})$, and nanoshell (480-810 nm) [1]. As shown in Figure 1, the combined PTT and PTT processes using various lights having wavelength from UV to IR with associate nanogold shapes and photosensitizer. Photosensitizer riboflavin (B2), 5-ALA, methylene blue (MB) and indocyanine green absorb, respectively, light at wavelength of $(365,430 \mathrm{~nm}),(530-670 \mathrm{~nm}),(780-850 \mathrm{~nm})$, as shown by Figure $2[1,10]$. Therefore, a combined dual-function of PTT/PDT can be performed by: (i) an NIR light at NIR absorbed by gold nanorod and indocyanine green; or a visible light absorbed by gold nanosphere and 5-ALA; (b) two different lights having wavelength at NIR (for PTT) and UV to visible light (for PDT). For the case of one light for both PTT and PDT the simultaneously interacting with the nanogold and the photosensitizer is much more complex that that of the case of two different lights which can be treated independently. We will start with the simpler case, where PTT and PDT will be modelled by the heat diffusion equation and the kinetic equation, respectively, as follows.

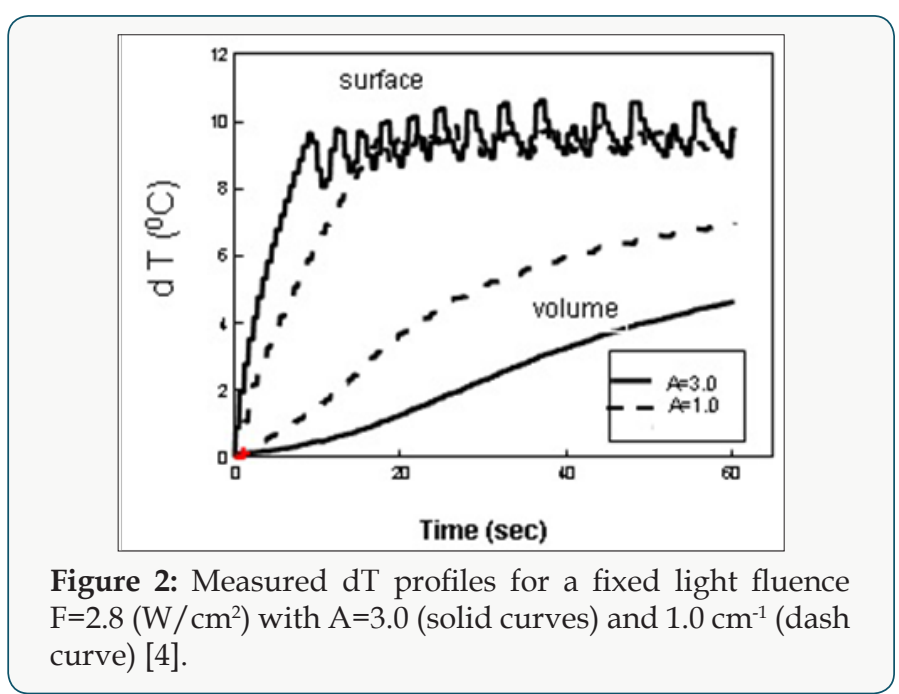

\section{The Temperature Increase in PTT}

The temperature profile of the laser irradiated GNR solutions may be solved numerically by a heat diffusion equation given by [4]

$$
\begin{gathered}
\frac{\partial^{2} T}{\partial z^{2}}-\frac{1}{K} \frac{\partial T}{\partial t}=-G(z) \\
G(z)=\left(\frac{A I}{K}\right) e^{-B Z}
\end{gathered}
$$

where $\mathrm{z}$ is the laser propagation direction along the depth of the GNRs solution, $\mathrm{k}$ and $\mathrm{K}$ are, respectively, the thermal conductivity and diffusivity of the solution, I is the laser intensity (or power density), $\mathrm{B}$ is the extinction coefficient, which can be expressed by $B=[A(A+2 s)]^{1 / 2}$, where $\mathrm{A}$ and $\mathrm{s}$ are the absorption and scattering coefficient. In this study we will assume that the scattering is much smaller than the absorption, and $\mathrm{B}=\mathrm{A}$. We note that $\mathrm{A}$ is proportional to the product of the extinction coefficient and concentration of the GNRs.

\section{Optimal conditions in PTT}

The light source terms $\mathrm{G}(\mathrm{z})$ have an optimal value. By taking $d G / d A\left(A=A^{*}\right)=0$, one obtains the maximal $G^{*}=I /(z K)$ under the optimal condition $A^{*}=1 / z$. The maximal $\mathrm{G}^{*}$ is resulted from the competing function of $\mathrm{A}$ and $\exp (-\mathrm{Bz})$. The optimal $\mathrm{A}^{*}$ also indicates that there is an optimal GNR concentration, $\mathrm{C}^{*}$, since A is proportional to $\mathrm{C}$. Lin et al proposed a novel pulsed-train method (PTM) to overcome the surface overheating issue (in conventional CW mode) and improve the volume temperature increase which is required for large volume tumors [4]. In our laboratory test, we 
demonstrated that the temperature increase profiles (dT). SurfacedT about 100C by the pulsed train on-off technique such that the volume-dT (at $\mathrm{z}=5.0 \mathrm{~mm}$ ) reaches about $80 \mathrm{C}$, which cannot be achieved in CW mode operation without overheating the solution surface. In addition, higher laser intensity shows faster surface-dT rising profile and higher volume-dT inside the solution (Figure 2).

We note that the PTM along cannot increase the volume-dT to the desired value. One shall also require an optimal A value (say about $1.0 \mathrm{~cm}^{-1}$ to $1.5 \mathrm{~cm}^{-1}$ ) which may be controlled either by the GNRs concentration or by using specific off-resonance laser wavelengths. Moreover, the A-value cannot be too small (say $<0.5 \mathrm{~cm}^{-1}$ ) which will require a longer time needed for a surface-dT reaching $10 \mathrm{OC}$. The novel features demonstrated in the above described also imply that cancer tumor having a dimension of $10 \times 10 \mathrm{~mm}$ can be treated using the PTM, but not by the conventional single pulse method Figure 2. Shows the measured surface and volume dT profiles for a fixed light fluence $\mathrm{F}=2.8\left(\mathrm{~W} / \mathrm{cm}^{2}\right)$ with $\mathrm{A}=3.0$ (solid curves) and $1.0 \mathrm{~cm}^{-1}$ (dash curve), where the smaller $A$ has higher volume temperature [4].

The in vivo situation in animal and/or human cancer therapy will be much more complex than the in vitro, simplified conditions described in above cancer cell model. These complexities shall include the non-uniform GNRs concentration in the tumor, the multi-layer normal-cancer tissue medium with multiple thermal parameters, and the blood flowing of the laser-targeted areas. In addition, the design of multiple-wavelengths laser system shall partially overcome the issues of GNRs non-uniform and multiple thermal medium for a 3-dimensional-therapy, in which various absorption penetration depths are available via the fiber-coupled multiple-wavelength laser simultaneously targeting the cancer tumors, The novel PTM and the laser system with auto-temperature control may provide useful tool for animal studies, where a fast temperature response time given by an IR camera may be integrated for real-time surface temperature monitoring.

\section{The combined PTT/PDT efficacy}

PDT process utilizes reactive oxygen species (ROS) generated through the reaction between photosensitizer (PS) and oxygen presented in tissues upon the irradiation of light to achieve effective treatment. The ROS is generated under a so-called typeII photochemical reaction which requires oxygen. In comparison, type-I process does not need oxygen and the triplet PS state can interact directly with the target for anti-cancer.

Using the same light for both the PS and nanogold interaction is much more complex than when two light with different wavelengths are absorbed respectively by the PS and nanogold, in which the PTT and PDT can be treated independently. Combining PTT and PDT using a single light, the kinetic equations of the light intensity $I(z, t)$, the PS concentration $\mathrm{C}(\mathrm{z}, \mathrm{t})$ and oxygen concentration, $\left[{ }^{3} \mathrm{O}_{2}\right]=\mathrm{Y}$, are given by [3]

$$
\frac{\partial c(z, t)}{\partial t}=-b[g=s K G] C
$$

$$
\begin{gathered}
\frac{\partial Y}{\partial t}=-b s G+P \\
\frac{\partial I(z, t)}{\partial z}=-A^{\prime}(z, t) I(z, t)
\end{gathered}
$$

Where $\mathrm{b}=\mathrm{aqI}(\mathrm{z}, \mathrm{t})$, UV intensity $\mathrm{I}(\mathrm{z}, \mathrm{t})$ in $\mathrm{mW} / \mathrm{cm}^{2}$; $\mathrm{q}$ is the PS triplet state [T] quantum yield; $g=\left(k_{8} / k_{3}\right)[A] G_{0} ; K=1 /(1+C+0.65[A])$, $G=C Y G_{0}$, with $G_{0}=1 /(Y+k) k=k_{5} / k_{3}+\left(k_{8} / k_{3}\right),[\mathrm{A}]$. The oxygen supply term is given by $P=P_{0}\left(1-Y /\left[0_{0}\right]\right),\left[O_{0}\right]$ being the initial oxygen concentration. Parameter $(\mathrm{N}=10)$ is added to fit the measured data of oxygen timeprofiles3. $s=s 1+s 2$ with $s 1$ and $s 2$ are the fraction of $\left[{ }^{3} \mathrm{O}_{2}\right]$ interacting with [T] to produce singlet oxygen (in type-II) and other ROS (in type-I), respectively. In Eq. (2.c), we have defined a new effective coefficient $A^{\prime}(z, t)=2.3\left[(a-b) C(z, t)+b C \_0+A+Q\right], a^{\prime}=a p$ with $p$ being the quantum yield for triplet PS state; $Q$ is the tissue absorption coefficient without nanogold or PS; A is the absorption constant of the nanogold; $a$ and $b$ are the extinction coefficient of the PS and the photolysis product having an concentration $\mathrm{C}(\mathrm{z}, \mathrm{t})$ with initial value $\mathrm{C}_{0}$. Eq. (2.b) also includes the light intensity reduction due to the absorption by nanogold via the $\exp (-\mathrm{Az})$ term of Eq. (1.b) when the same light is used for both PTT and PDT. We note that stronger PTT (or larger Az) produces higher temperature, which however, also reduces the available light intensity for PDT. Therefore, there is an optimal condition depending upon either PTT or PDT will be the dominated process for optimal clinical outcomes.

\section{Optimal efficacy in PDT}

For the PDT dominant case, both type-I and type-II reactions occur in the photochemical reaction. The kinetic equation of the the photoinitiation rate for type-I $\left(R_{1}\right)$ process and type-II $\left(R_{2}\right)$ generation of the reactive oxygen species (ROS) is given by The anti-cancer efficacy is related to the S-function by Ceff=1-exp(-S), where S1 (for type-I) and S2 (for type-II) are given by [3]

$$
\begin{gathered}
S 1=E^{\prime} \sqrt{4 K^{\prime} C_{0} F(z) \exp \left(A^{\prime} z\right) /\left(a q I_{0}\right)} \\
S 2=b \int_{0}^{t} G(z, t) /[A] d t
\end{gathered}
$$

with $E^{\prime}(z, t)=[1-\exp (-0.5 b t)], K^{\prime}$ is an effective rate constant.

Eq. (2) and (3) can be solved only numerically. For the case for PDT only, or when PTT is neglected, the Az term in A' of Eq. (2.c) can be neglected. We have numerically showed that S1 has an optimal $\mathrm{z}^{*}$, whereas S2 is a decreasing function of $\mathrm{z}$, and achieves a steady state in time. It was reported that type-II, or S2, is the predominant process for anti-cancer, which is governed by the oxygen concentration. S2 reaches a steady state in time when oxygen is completely depleted.

\section{Optimal synergic effects}

As shown by Eq. (3), the $S$ formulas show that $S 1 \sim\left[C_{0} / I_{0}\right]^{0.5}$, with no contribution from oxygen $\left[\mathrm{O}_{2}\right]$; whereas $S 2 \sim\left[\mathrm{O}_{2}\right] \mathrm{C}$ requires both $\mathrm{C}$ and $\left[\mathrm{O}_{2}\right]$. Therefore, resupply of PS or oxygen would improve the generation of ROS, and improve the anti-cancer efficacy via typeII PDT. Figure 3 shows profiles of oxygen and PS concentration, $\left[\mathrm{O}_{2}\right]$ 
(red curves) and C (blue curves), for various light intensity of 50 , $100,200 \mathrm{~mW} / \mathrm{cm}^{2}$, with external oxygen source $\mathrm{P}>0$, which would improve the type-II efficacy (or S2 function) (Figure 3).

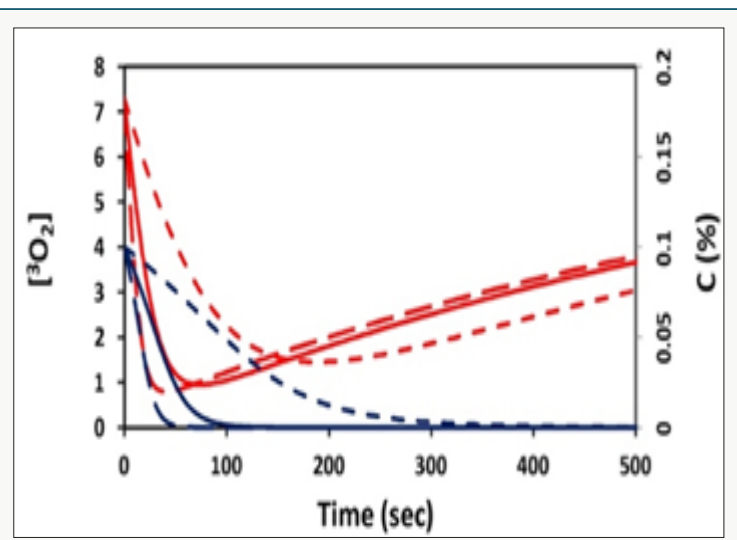

Figure 3: Profiles of oxygen and PS concentration, $\left[\mathrm{O}_{2}\right]$ (red curves) and $C$ (blue curves), for various light intensity of $50,100,200 \mathrm{~mW} / \mathrm{cm}^{2}$ (for curves in dot, solid and dash).

Eq. (2) and (3) show the following important features for the PDT efficacy defined by the amount of ROS generation. S2 is an increasing function of the light energy (or intensity $x$ time) and its quantum yield (q).

However, it is reduced by the PS concentration depletion (with a quantum yield p). For maximum type-II PDT efficacy one requires the following conditions: large q (or high quantum yield), sufficient oxygen supply during the reaction, with $\mathrm{P}>0$ ), small A (or small absorption by the nanogold). On the other hand, for high PTT efficacy one requires large temperature increase (dT) which is proportional to the light absorption in the nanogold, or $\mathrm{AI}_{0}$. Therefore, in case-1 using one light to perform both PTT and PDT would require higher light energy (or intensity) comparing to case-2, using two different lights for PTT and PDT, independently without co-absorption, and therefore PTT and PDT can be treated separately. In case-1, the system design is simpler and cost effective. However, the collective effects between PTT and PDT require complex optimization of the concentration of PS and nanogold, besides that the wavelength of the light must match both the absorption peak of PS and nanogold. Greater details requiring numerical solutions of Eq. (3) and (5) will be presented elsewhere.

The synergic effects of PTT and PDT, using two lasers at 808 $\mathrm{nm}$ and $660 \mathrm{~nm}$, respectively, and nanogold in C6 gel, was reported by Kim et al. [10]. They reported higher efficacy when conduct PDT prior to PTT than [PTT+PDT]. This sequential-dependent process may be realized by that PTT may cause reduction of the kinetic constant and quantum yield due to increased temperature due to PTT, besides the potential reduction of oxygen supply which is critical in type-II PDT. In addition to the methods presented in this paper, the efficacy of PDT may be further improved significantly via conjugated nanogolds. For example, it was reported by the conjugated spherical nanogold as the delivery agent for 5-ALA resulted in a two times higher cell death rate compared to free
5-ALA [11]. Another example is that the DNA damage caused by PDT as demonstrated by alkaline gel electrophoresis was greater in the methylene blue (MB) plus chitosan-treated group than in control and MB-treated groups [12-13].

\section{Conclusion}

Efficacy of cancer therapy may be enhanced by combining PTT and PDT either activated by one light or two lights. For maximum PTT/PDT synergistic efficacy, the concentration of PS and nanogold required optimization, besides the wavelength of the light matching the absorption peak of PS and nanogold, and the order of PTT and PDT process. External supply of either PS or oxygen concentration will significantly improve the anti-cancer efficacy via type-II PDT, which is limited by the generation of ROS, or the depletion of oxygen and/or PS concentration.

\section{Acknowledgment}

This work was supported by the internal grant of New Vision Inc. KT Chen is partially supported by the Ph.D program of Graduate Institute of Applied Science and Engineering, Fu Jen Catholic University, Taiwan.

\section{References}

1. Lin JT, Chen KT, Liu HW (2017) Progress of nanotechnology for phototherapy: Fundamentals and Applications. Med Devices Diagn Eng.

2. Lin JT (2017) Analysis of the efficiency of photothermal and photodynamic cancer therapy via nanogolds and photosensitizers. J Cancer Research update 6(1): 12-18.

3. Lin JT, Chen KT, Liu HW (2018) Analysis of kinetics and efficacy of anticancer via oxygen-enhanced photodynamic therapy. J Cancer Research update $7(1): 21-26$.

4. Jui-Teng Lin, Yueh-Sheng Chiang, Guang-Hong Lin, Hsinyu Lee, Hsia-Wei Liu (2012) In vitro photothermal destruction of cancer cells using gold nanorods and pulsed-train near-infrared lase. J Nanomaterials.

5. Huang X, El Sayed MA (2010) Gold nanoparticles: Optical properties and implementations in cancer diagnosis and photothermal therapy. J Advanced Research 1(1): 13-28.

6. Popp MK, Imane Oubou, Colin Shepherd, Zachary Nager, Courtney Anderson, et al. (2014) Photothermal therapy using gold nanorods and near-infrared light in a murine melanoma model increases survival and decreases tumor. J nanomaterials.

7. Lin JT (2011) Nonlinear optical theory and figure of merit of surface plasmon resonance of gold nanorods. J Nanophotonics 5(1): 051506.

8. Lin JT (2010) Scaling law and figure of merit of biosensor using gold nanoshells. J Nanophotonics 4: 049507.

9. Lin JT (2013) A generalized geometric factor for unified scaling law for bio-sensor based on nanostructures of gold. Inter J Latest Res Science and Tech 2(6): 23-25.

10. Kim JY, Choi W, Kim M, Tae G (2013) Tumor-targeting nanogel that can function independently for both photodynamic and photothermal therapy and its synergy from the procedure of PDT followed by PTT. Journal of Controlled Release 171(2): 113-121.

11. Shemesh CS, Hardy CW, Yu DS, Fernandez B, Zhang H (2014) Indocyanine green loaded liposome nanocarriers for photodynamic therapy using human triple negative breast cancer cells. Photodiag Photodany Therapy 
11(2): 193-203.

12. Akens MK, Wise Milestone L, Won E, Schwock J, Yee AJ, et al. (2014) In vitro and in vivo effects of photodynamic therapy on metastatic breast cancer cells pre-treated with zoledronic acid. Photodiag Photodany Therapy 11(3): 422-433.
13. Mohammadi Z, Sazgarnia A, Rajabi O, Soudmand S, Esmaily H, et al. (2013) An in vitro study on the photosensitivity of 5-aminolevulinic acid conjugated gold nanoparticles. Photodiag Photodany Therapy 10(4): 382-388.

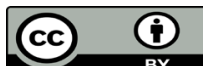

This work is licensed under Creative Commons Attribution 4.0 License

To Submit Your Article Click Here:

Submit Article

DOI: $10.32474 /$ OAJBEB.2018.02.000126

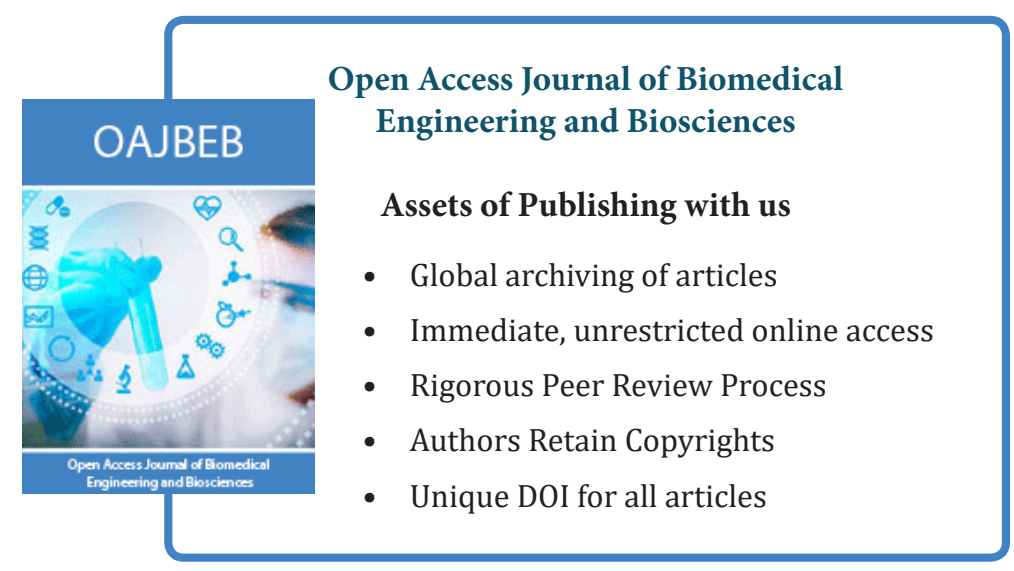

